\title{
Topological minimal self-joinings
}

\author{
NELSON G. MARKLEY \\ Department of Mathematics, University of Maryland, College Park, MD 20742, USA
}

(Received 22 October 1982 and revised 20 January 1983)

\begin{abstract}
There is an interesting duality between some of the concepts of ergodic theory and those of topological dynamics. This paper is a first attempt at developing a topological analogue to the measure-theoretic notion of a transformation having minimal self-joinings. The main problem is to understand the dynamics of the composition of a cartesian product of powers of a transformation having topological minimal self-joinings with a compact permutation of the coordinates. Most of the results are about the minimal subsets of such a composition.
\end{abstract}

\section{Introduction}

Rudolph [9] introduced the concept of minimal self-joinings (MSJ) to build examples of exotic ergodic behaviour. His example of MSJ was not in the context of a concrete homeomorphism of a familiar compact metric space. Since then del Junco, Rahe and Swanson [3] have shown that Chacon's example [1] has MSJ. This example can be constructed by 'doubling the ones' in a certain symbolic almost automorphic minimal set whose maximal equicontinuous factor is the 3 -adic integers. This construction is analogous to that used by Furstenberg, Keynes and Shapiro [5] to construct a proximal orbit dense (POD) flow. In fact, del Junco [2] has pointed out that Chacon's example is a POD flow. (The author was also aware of the existence of POD flows similar to the Chacon example [8].)

These developments raise two natural questions. What is the relationship between MSJ and POD? What is the topological analogue of MSJ? Del Junco [2] has partially answered the first question. This paper is devoted to the second question and proceeds from the premise that minimal sets should be analogous to ergodic measures. This produces an interesting theory, but the results thus far are not as good as one might desire.

The main thrust of our work is to study the dynamics of the obvious maps which commute with a countable power of a system with topological minimal self-joinings. In other words, we investigate Rudolph's $U(\pi, l)$ maps from a topological rather than measure theoretic viewpoint. Moreover, our analysis focuses on the minimal subsets of these maps.

\section{Preliminaries}

Let $X$ be a compact metric space. Given a homeomorphsim $T$ of $X$ onto itself, the pair $(X, T)$ will be called a flow. The flow $(X, T)$ is (topologically) ergodic if every proper closed invariant set is nowhere dense, and (topologically) weak mixing if 
$(X \times X, T \times T)$ is ergodic. The flow $(X, T)$ is point transitive if there exists a point $x \in X$ whose orbit $\left\{T^{n} x: n \in \mathbb{Z}\right\}$ is dense in $X$. (As usual $\mathbb{Z}$ denotes the integers.) Because $X$ is metric the Baire category theorem can be used to show that weak mixing implies point transitive. A stronger notion than ergodicity is minimality which means there are no proper closed invariant subsets. Equivalently a flow is minimal if every orbit is dense. A minimal subset of $(X, T)$ is, of course, a closed invariant subset $M$ of $X$ such that $(M, T \mid M)$ is a minimal flow. The flow $(X, T)$ is totally minimal if $\left(X, T^{n}\right)$ is minimal for all $n \in \mathbb{Z}, n \neq 0$. Keynes has shown that a flow which is both weak mixing and minimal is totally minimal [6, theorem 3.1].

A homomorphism from $(X, T)$ to $(Y, S)$ is a continuous map $\varphi$ from $X$ to $Y$ such that $\varphi T=S \varphi$. The terms isomorphism, automorphism, and endomorphism will be used in the usual way. We will say $(Y, S)$ is a factor of $(X, T)$ or $(X, T)$ is an extension of $(Y, S)$ if there exists an onto homomorphism from $(X, T)$ to $(Y, S)$. If $\varphi$ is a homomorphism of $(X, T)$ onto $(Y, S)$, then

$$
R=\left\{\left(x_{1}, x_{2}\right) \in X \times X: \varphi\left(x_{1}\right)=\varphi\left(x_{2}\right)\right\}
$$

is a closed invariant equivalence relation on $X$. Conversely, any closed invariant equivalence relation on $X$ determines a factor $(X / R, S)$ of $(X, T)$. We will say a factor is proper if the corresponding closed invariant equivalence relation is not the diagonal

$$
\Delta=\left\{\left(x_{1}, x_{2}\right) \in X \times X: x_{1}=x_{2}\right\} .
$$

The proximal relation $P$ for a flow $(X, T)$ is the set of all points $(x, y) \in X \times X$ such that there exists a sequence of integers $\left\{k_{i}\right\}$ for which

$$
\lim _{i \rightarrow \infty} T^{k_{i}} x=\lim _{i \rightarrow \infty} T^{k_{i}} y .
$$

We say $x$ and $y$ are proximal when $(x, y) \in P$ and distal when $(x, y) \notin P$. Suppose $(Y, S)$ is a factor of $(X, T)$ determined by the closed invariant equivalence relation $R$ on $X$. Then $(X, T)$ is called a proximal extension if $R \subset P$ and a distal extension if $R \cap P=\Delta$.

One other kind of extension will occur in this paper. Suppose $G$ is a compact topological group acting continuously on $X$ such that $g T=T g$ for all $g \in G$. Then

$$
R=\left\{\left(x_{1}, x_{2}\right) ; x_{2}=g x_{1} \quad \text { for some } g \in G\right\}
$$

is a closed invariant equivalence relation on $X$. We will call $(X, T)$ a group extension of $(X / R, S)$. Note that any finite group of automorphisms determines a group extension.

Since we will be working primarily with cartesian products of powers of $T: X \rightarrow X$, it will be convenient to have some simple notation for handling them.

Let $D$ be a countable set (finite or infinite). Then each $\alpha \in \mathbb{Z}^{D}$ defines a flow $\left(X^{D}, \alpha\right)$ by

$$
(\alpha x)(d)=T^{\alpha(d)} x(d) .
$$

Here we are thinking of $\alpha \in \mathbb{Z}^{D}$ and $x \in X^{D}$ as functions from $D$ into $\mathbb{Z}$ and $X$ respectively. In this notation $T^{n} x$ would be $n x$ and $\left(X \times X, T \times T^{3}\right)$ would be 
$\left(X^{2},(1,3)\right.$ ). (When $F$ is finite with cardinality $k$, we assume $D=\{1,2, \ldots, k\}$ and write $X^{k}$ instead of $X^{D}$.)

Proposition 1.1. Suppose $(X, T)$ is weak mixing and minimal. Then $\left(X^{D}, \alpha\right)$ is weak mixing if and only if $\alpha(d) \neq 0$ for all $d \in D$.

Proof. If some $\alpha(d)=0$, then obviously there exist proper open invariant sets.

Assume $\alpha(d) \neq 0$ for all $d \in D$ and $D$ is finite. Then $\left(X, T^{n}\right)$ is weak mixing for $n \neq 0\left[6\right.$, theorem 2.6], and by repeated applications of lemma 5.1 of $[6],\left(X^{D}, \alpha\right)$ is weak mixing. The infinite case follows easily from the finite case.

Corollary 1.2. Let $(X, T)$ be a weak mixing minimal flow. Then for $D$ countable $\left(X^{D}, \alpha\right)$ is point transitive if and only if $\alpha(d) \neq 0$ for all $d \in D$.

\section{Elementary properties of TMSJ}

For each $\alpha \in \mathbb{Z}^{D}$ there is a natural embedding of $(X, T)$ in $\left(X^{D}, \overline{1}\right)$ where $\overline{1}(d)=1$ for all $d$ in $D$. Define the diagonal by

$$
\Delta=\left\{x \in X^{D}: x(d)=x\left(d^{\prime}\right) \text { for all } d, d^{\prime} \in D\right\}
$$

and set $\Gamma(\alpha)=\alpha \Delta$, which is called an off-diagonal. In other words, $x \in \Gamma(\alpha)$ if and only if there exists $y \in X$ such that $x(d)=T^{\alpha(d)} y$ for all $d$. Clearly $\Gamma(\alpha)$ is closed and invariant under $\overline{1}$. Alternatively, the off-diagonals can be thought of as graphs, for example, when $\alpha=(0, k) \in \mathbb{Z}^{2}$, then $\Gamma(\alpha)$ is just the graph of $T^{k}$. When $k \in \mathbb{Z}$, we set $\Gamma(k)=X$.

Proposition 2.1. Suppose $(X, T)$ has at least one infinite orbit, and let $\alpha, \beta \in \mathbb{Z}^{D}$. Then the following hold:

(a) $\Gamma(\alpha)=\Gamma(\beta)$ if and only if there exists an integer $k$ such that $\alpha=\beta+k \overline{1}$;

(b) $\alpha \Gamma(\beta)=\Gamma(\beta)$ if and only if $\alpha=k \overline{1}$.

Proof. (a) Let $y \in X$ such that $T^{n} y \neq y$ for all $n \neq 0$. Suppose there exists $y^{\prime} \in X$ such that $T^{\alpha(d)} y=T^{\beta(d)} y^{\prime}$ for all $d \in D$. It follows that

$$
[\alpha(d)-\beta(d)]-\left[\alpha\left(d^{\prime}\right)-\beta\left(d^{\prime}\right)\right]=0
$$

for all $d, d^{\prime} \in D$. Let $k$ be the common value for $\alpha(d)-\beta(d)$. The converse is obvious.

(b) Note that $\alpha \Gamma(\beta)=\Gamma(\alpha+\beta)$ and use (a).

Clearly $(\Gamma(\beta), k \overline{1})$ is always isomorphic to $\left(X, T^{k}\right)$. We want a similar fact for any $\alpha \in \mathbb{Z}^{D}$ instead of $k \overline{1}$. Let $\Lambda \subset \mathbb{Z}$ be the range of $\alpha$ and for $j \in \Lambda$ set $D_{j}=\alpha^{-1}(j)$, which partitions $D$. Define $\lambda \in \mathbb{Z}^{\Lambda}$ by $\lambda(j)=j$. Then for any $\boldsymbol{\beta}_{j} \in \mathbb{Z}^{D_{j}}, j \in \Lambda,\left(X^{\Lambda}, \lambda\right)$ is isomorphic to the flow

$$
\left(\prod_{j \in \Lambda} \Gamma\left(\beta_{j}\right), \alpha\right)
$$

where $\prod_{j \in \Lambda} \Gamma\left(\beta_{j}\right)$ is, in the natural way, viewed as a subset of $X^{D}$. We will refer to $\left\{D_{j}\right\}_{j \in \Lambda}$ as the canonical partition determined by $\alpha$ and to $\lambda$ as the canonical injection determined by $\alpha$.

Proposition 2.2. Let $(X, T)$ be a minimal non-periodic flow, and let $\alpha \in \mathbb{Z}^{D}$. Suppose $\left\{D_{j}\right\}_{j \in \Lambda}$ is a partition of $D$ and $\beta_{j} \in \mathbb{Z}^{D_{j}}$ for $j \in \Lambda$ such that $\prod_{j \in \Lambda} \Gamma\left(\beta_{j}\right)$ is a minimal 
subset of $\left(X^{D}, \alpha\right)$. Then $\left\{D_{j}\right\}_{j \in \Lambda}$ is the canonical partition determined by $\alpha$ and $\left(X^{\Lambda}, \lambda\right)$ is minimal, where $\lambda$ is the canonical injection determined by $\alpha$. Moreover, $\alpha(d) \neq 0$ for all $d \in D$.

Proof. Let $\alpha_{j}=\alpha \mid D_{j}$. Then $\alpha\left(\prod_{j \in \Lambda} \Gamma\left(\beta_{j}\right)\right)=\prod_{j \in \Lambda} \Gamma\left(\beta_{j}\right)$ implies $\alpha_{j} \Gamma\left(\beta_{j}\right)=\Gamma\left(\beta_{j}\right)$ and $\alpha_{j}=k_{j} \overline{1}, k_{j} \in \mathbb{Z}$. Thus $\alpha(d)=\alpha\left(d^{\prime}\right)$ for all $d, d^{\prime} \in D_{j}$. If $k_{j}=k_{i}$ for $j \neq i$, then $\prod_{j \in \Lambda} \Gamma\left(\beta_{j}\right)$ is not minimal because $\left(X^{2},(k, k)\right)$ is never minimal. The rest is obvious.

Suppose $(X, T)$ has a unique invariant measure $\mu$, and $T$ has minimal self-joinings when viewed as a measure preserving transformation. Roughly speaking, minimal self-joinings means every invariant measure for $\left(X^{D}, \alpha\right), \alpha(d) \neq 0$ for all $d$, with all its marginals equal to $\mu$ is a product of the natural copies of $\mu$ on off-diagonals. (See the introduction to [9].) Let $M$ be a minimal subset of $\left(X^{D}, \alpha\right)$ where $\alpha(d) \neq 0$ for all $d \in D$. There exists an ergodic invariant measure $\nu$ for $\left(X^{D}, \alpha\right)$ with support $M$. Clearly, the marginals of $\nu$ are all $\mu$. Therefore, $\nu$ is the product of off-diagonal measures. In particular, there exists a partition $\left\{D_{j}\right\}_{j \in \Lambda}$ of $D$ and $\beta_{j} \in \mathbb{Z}^{D_{i}}$ such that

$$
M=\prod_{j \in \Lambda} \Gamma\left(\beta_{j}\right)
$$

This suggests the following definition: the flow $(X, T)$ has topological minimal self-joinings (TMSJ) if $(X, T)$ is (topologically) weak mixing and given $\alpha \in \mathbb{Z}^{D}$ with $D$ countable and $\alpha(d) \neq 0$ for all $d \in D$, then every minimal subset $M$ of $\left(X^{D}, \alpha\right)$ is of the form

$$
M=\prod_{j \in \Lambda} \Gamma\left(\beta_{j}\right)
$$

for some partition $\left\{D_{j}\right\}_{j \in \Lambda}$ of $D$ and $\beta_{j} \in \mathbb{Z}^{D_{j}}$. When the above holds only under the more restrictive condition that $\alpha(d)>0$ for all $d$, we add the adjective 'positive' (PTMSJ). Note TMSJ and PTMSJ imply $(X, T)$ is minimal and TMSJ implies PTMSJ.

Proposition 2.3. Suppose $(X, T)$ has TMSJ \{PTMSJ\} and $\alpha \in \mathbb{Z}^{D}$ with $\alpha(d) \neq 0$ $\{\alpha(d)>0\}$ for all $d \in D$. The following are equivalent:

(a) $\alpha$ is injective, i.e. $\alpha(d) \neq \alpha\left(d^{\prime}\right)$ for all $d \neq d^{\prime}$;

(b) $\left(X^{D}, \alpha\right)$ is minimal;

(c) $\left(X^{D}, \alpha\right)$ is totally minimal.

Proof. Use the definitions and proposition 2.2.

Proposition 2.4. Suppose $(X, T)$ has TMSJ $\left\{\right.$ PTMSJ\} and $\alpha \in \mathbb{Z}^{D}$ with $\alpha(d) \neq 0$ $\{\alpha(d)>0\}$ for all $d \in D$. If $\varphi$ is an endomorphism of $\left(X^{D}, \alpha\right)$ and $\left(X^{D}, \alpha\right)$ is minimal, then $\varphi=\beta$ for some $\beta$ in $\mathbb{Z}^{D}$. In particular, if $\varphi$ is an endomorphism of $(X, T)$, then $\varphi=T^{n}$ for some $n \in \mathbb{Z}$.

Proof. Consider the minimal set $M=\left\{(x, \varphi x): x \in X^{D}\right\}$, and apply proposition 2.2. to $\left(X^{D} \times X^{D}, \alpha \times \alpha\right)$.

It is also easy to prove the following:

Proposition 2.5. If $(X, T)$ has TMSJ $\{$ PTMSJ\}, then every factor of $(X, T)$ has TMSJ \{PTMSJ . 
A flow $(X, T)$ is called a proximal orbit dense (POD) flow if $(X, T)$ is totally minimal and whenever $x, y \in X$ with $x \neq y$, then for some $n \neq 0, x$ and $T^{n} y$ are proximal. Equivalently, $(X, T)$ is a POD flow if it is totally minimal and when $x \neq y$,

$$
\left.\mathrm{Cl}\left\{T^{n} x, T^{n} y\right): n \in \mathbb{Z}\right\} \supset \Gamma(0, k)
$$

for some $k \neq 0$. It follows that when $(X, T)$ is a POD flow the minimal subsets of $(X \times X, T \times T)$ are precisely the sets $\Gamma(\alpha), \alpha \in \mathbb{Z}^{2}$; this concept is due to Furstenberg. Keynes and Shapiro [5] who showed that POD flows are prime, i.e. if $(Y, S)$ is a factor of a POD flow $(X, T)$, then $(Y, T)$ is either isomorphic to $(X, T)$ or is the trivial minimal flow on a single point. They also showed that if $(X, T)$ is a POD flow, then either $(X, T)$ is isomorphic to $\left(X, T^{-1}\right)$ or

$$
\left(X^{2},(1,-1)\right)=\left(X \times X, T \times T^{-1}\right)
$$

is minimal. Moreover, it is known that POD flows must occur on metric spaces [6, lemma 2.1 and theorem 2.2].

THEOREM 2.6. If $(X, T)$ is a POD flow, then $(X, T)$ has PTMSJ or TMSJ according to whether $(X, T)$ is or is not isomorphic to $\left(X, T^{-1}\right)$.

Proof. Let $\alpha$ be an element of $\mathbb{Z}^{D}$ satisfying the required conditions. Let $\Lambda$ and $\lambda$ denote the canonical decomposition and injection determined by $\alpha$. Then $\left(X^{\Lambda}, \lambda\right)$ is minimal [7, corollary 3.6].

Consider $M$ a minimal subset of $\left(X^{D}, \alpha\right)$ and let $\pi_{j}$ be the projection of $X^{D}$ onto $X^{D_{j}}$ for each $j \in \Lambda$. Then $\pi_{j}(M)$ is a minimal subset of $\left(X^{D_{j}}, \lambda(j) \overline{1}\right)$ and

$$
M \subset \prod_{j \in \Lambda} \pi_{j}(M)
$$

The next proposition will show that each $\pi_{j}(M)=\Gamma\left(\beta_{j}\right)$ for some $\beta_{j} \in \mathbb{Z}^{D_{j}}$. Since $\left(\prod_{j \in \Lambda} \Gamma\left(\beta_{j}\right), \alpha\right)$ is isomorphic to the minimal flow $\left(X^{\Lambda}, \lambda\right)$, it will then follow that

$$
M=\prod_{j \in \Lambda} \Gamma\left(\beta_{j}\right) .
$$

Proposition 2.7. Let $(X, T)$ be a totally minimal flow. If the only minimal sets of $\left(X^{2}, \overline{1}\right)=(X \times X, T \times T)$ are the sets $\Gamma(\alpha), \alpha \in \mathbb{Z}^{2}$, then the only minimal subsets of $\left(X^{D}, m \overline{1}\right), D$ countable and $m \neq 0$, are the sets $\Gamma(\alpha), \alpha \in \mathbb{Z}^{D}$.

Proof. First let $M$ be a minimal subset of $\left(X^{2}, m \overline{1}\right)$. We can assume $m>1$. Set

$$
M^{\prime}=\bigcup_{i=0}^{m-1}(i \overline{1}) M .
$$

Then $M^{\prime}$ is a closed invariant set under $\overline{1}$ and contains some minimal set $\Gamma(\alpha)$. Because $(X, T)$ is totally minimal, $\Gamma(\alpha)$ is also a minimal subset of $\left(X^{2}, m \overline{1}\right)$. Thus $\Gamma(\alpha)=(i \overline{1}) M$ for some $i, 0 \leq i \leq m-1$ from which it follows that $M=\Gamma(\alpha)$.

Now let $M$ be a minimal subset of $\left(X^{D}, m \overline{1}\right)$. Pick $d_{0} \in D$. For each $d \in D, d \neq d_{0}$, let $\pi_{d}$ be the projection of $X^{D}$ onto $X^{2}$ defined by

$$
\pi_{d}(x)=\left(x\left(d_{0}\right), x(d)\right) .
$$

Clearly, $\pi_{d}(M)$ is a minimal subset of $\left(X^{2}, m \overrightarrow{1}\right)$ and there exists $\alpha(d) \in \mathbb{Z}$ such that

$$
\pi_{d}(M)=\{(x, \alpha(d) x): x \in X\} .
$$


Setting $\alpha\left(d_{0}\right)=0$, we have $M \subset \Gamma(\alpha)$. Finally, $M=\Gamma(\alpha)$ because $\Gamma(\alpha)$ is a minimal subset of $\left(X^{D}, m \overline{1}\right)$.

Keynes and Newton showed that if $\varphi:(Y, S) \rightarrow(X, T)$ is an almost one-to-one homomorphism $\left(\varphi^{-1}(x)\right.$ is a singleton for at least one $\left.x\right)$ and $(X, T)$ is POD, then $\left(Y^{2}, \overline{1}\right)$ has only off-diagonals as minimal subsets [7, lemma 2.16$]$. The next proposition gives a slightly stronger result.

Proposition 2.8. If $(Y, S)$ is minimal, $\varphi:(Y, S) \rightarrow(X, T)$ is an almost one-to-one homomorphism and $(X, T)$ has TMSJ $\{\mathrm{PTMSJ}\}$, then $(Y, T)$ also has TMSJ $\{$ PTMSJ .

Proof. Let $A=\left\{x:\left|\varphi^{-1}(x)\right|=1\right\}$ and define $e: A \rightarrow Y$ by $e(x)=\varphi^{-1}(x)$. Then $A$ is a dense invariant subset of $X$ and $e$ is an isomorphism of $(A, T)$ into $(Y, S)$. It follows that $(Y, S)$ is totally minimal. Furthermore, if we define $\hat{\varphi}:\left(Y^{D}, \alpha\right) \rightarrow\left(X^{D}, \alpha\right)$ and $\hat{e}:\left(A^{D}, \alpha\right) \rightarrow\left(Y^{D}, \alpha\right)$ by $(\hat{\varphi} x)(d)=\varphi(x(d))$ etc., then this situation reoccurs and $\left(Y^{D}, \alpha\right)$ is minimal when $\left(X^{D}, \alpha\right)$ is minimal.

Let $M$ be a minimal subset of $\left(Y^{D}, \alpha\right)$. Then $\hat{\varphi}(M)=\prod_{i \in \Lambda} \Gamma_{X}\left(\beta_{i}\right)$. Since $\prod_{i \in \Lambda} \Gamma_{X}\left(\beta_{i}\right) \cap A^{D} \neq \varnothing$,

$$
M \subset \prod_{i \in \Lambda} \Gamma_{Y}\left(\beta_{i}\right) \cong\left(Y^{\Lambda}, \lambda\right),
$$

which is minimal because $\left(X^{\Lambda}, \lambda\right)$ is minimal.

Furstenberg, Keynes and Shapiro [5] constructed POD flows. Del Junco [2] has pointed out that for their examples $(X, T)$ is isomorphic to $\left(X, T^{-1}\right)$. Furthermore, del Junco's work [2] together with his joint work with Rahe and Swanson [3] shows that Chacon's example [1] has MSJ and is POD. Thus there are POD flows for which $(X, T)$ is not isomorphic to $\left(X, T^{-1}\right)$. Consequently TMSJ and PTMSJ are distinct notions which together generalize POD.

\section{3. $U(\pi, \alpha)$ minimal sets: finite order case}

Let $\pi$ be a permutation of $D$ where, as always, $D$ is countable, and let $(X, T)$ be a flow. Then

$$
S_{\pi} x(d)=x\left(\pi^{-1}(d)\right)
$$

defines a homeomorphism of $X^{D}$ onto itself and for each $\alpha \in \mathbb{Z}^{D}$,

$$
U=U(\pi, a)=S_{\pi} \circ \alpha
$$

defines an automorphism of $\left(X^{D}, \overline{1}\right)$. The general problem is to understand the dynamics of $\left(X^{D}, U\right)$ when $(X, T)$ has TMSJ.

We will need to assume that $\pi$ is compact, that is, for all $d \in D$ the cardinality $l(d)$ of the set $\left\{\pi^{k}(d): k \in \mathbb{Z}\right\}$ is finite. Even without any infinite cycles $\pi$ can still have infinite order in the group of all permutations on $D$, namely when $l(D)$ is not a finite subset of $\mathbb{Z}$. This determines two distinct cases. In this section we will always assume $\pi$ has finite order. 
Let $l(D)=\left\{a_{1}, a_{2}, \ldots, a_{r}\right\}$ and let $\rho$ equal the least common multiple of $a_{1}, \ldots, a_{r}$. So $\rho$ is the order of $\pi$. Define $\alpha_{\pi} \in \mathbb{Z}^{D}$ by

$$
\alpha_{\pi}(d)=\sum_{i=1}^{\rho} \alpha\left(\pi^{-i}(d)\right)
$$

Note that

$$
\alpha_{\pi}(d)=\frac{\rho}{l(d)} \sum_{i=1}^{l(d)} \alpha\left(\pi^{i}(d)\right)
$$

Clearly, $\left(X^{D}, U^{\rho}\right)$ is the same as $\left(X^{D}, \alpha_{\pi}\right)$.

Remark 3.1. Let $(X, T)$ be a weak mixing minimal flow. Then $\left(X^{D}, U(\pi, \alpha)\right)$ is weak mixing if and only if $\alpha_{\pi}(d) \neq 0$ for all $d \in D$.

Remark 3.2. Assume $X$ is not finite. The flow $\left(X^{D}, U(\pi, \alpha)\right)$ is minimal if and only if $\pi$ is the identity and $\left(X^{D}, \alpha\right)$ is minimal. Moreover, $M$ is a minimal subset of $\left(X^{D}, U(\pi, \alpha)\right)$ if and only if there exists a minimal subset $M_{0}$ of $\left(X^{D}, \alpha_{\pi}\right)$ such that

$$
M=\bigcup_{i=0}^{\rho-1} U^{i} M_{0}
$$

If $(X, T)$ has TMSJ $\{$ PTMSJ $\}$ and $\alpha_{m}(d) \neq 0\left\{\alpha_{n}(d)>0\right\}$ for all $d \in D$, then every minimal set of $\left(X^{D}, \alpha_{n}\right)$ is canonically isomorphic to $\left(X^{\Lambda}, \lambda\right)$ when $\lambda$ is the canonical injection determined by $\alpha_{\pi}$. Thus the minimal sets of $\left(X^{D}, U(\pi, \alpha)\right)$ are all constructed in some way from the same underlying minimal set. There is a natural general construction which we will use to analyze this situation.

For any flow $(X, T)$ there is a standard way to construct an extension of the cyclic flow on $p$ points using $(X, T)$. Let $X_{p}=X \times\{0,1, \ldots, p-1\}$ and define $T_{p}$ by

$$
T_{p}(x, i)= \begin{cases}(x, i+1) & \text { if } i<p-1 \\ (T x, 0) & \text { if } i=p-1 .\end{cases}
$$

If $(X, T)$ is totally minimal, then $\left(X_{p}, T_{p}\right)$ is minimal for all positive integers $p$.

For a specific flow $(X, T)$, let $\chi(p, q, \beta)$ denote the flow obtained by applying the above construction to $\left(X^{D}, q \beta\right)$. i.e.

$$
\chi(p, q, \beta)=\left(\left(X^{D}\right)_{p},(q \beta)_{p}\right) .
$$

In this notation $p$ and $q$ will always be positive integers, and $\beta$ will always be an element of $\mathbb{Z}^{D}$ for some countable $D$. In other words, for a given flow $(X, T)$, $\chi(p, q, \beta)$ describes a family of flows constructed from $(X, T)$ and parameterized by $p, q$, and $\beta$. The purpose of the seemingly superfluous $q$ is to remove common factors from the coordinates of $\beta$. In this notation

$$
\begin{aligned}
(X, T) & =\chi(1,1,1), \quad(X, \alpha)=\chi(1,1, \alpha), \\
\left(X^{2},\left(T \times T^{3}\right)^{2}\right) & =\chi(1,2,(1,3)) \quad \text { and } \quad\left(X_{p}, T_{p}\right)=\chi(p, 1,1),
\end{aligned}
$$

and

$$
\chi(p, q, \beta)=\chi(p, 1, q \beta) .
$$

Proposition 3.3. Assume $\left(X^{D}, \beta\right)$ is totally minimal. If $\chi\left(p^{\prime}, q^{\prime}, \beta\right)$ is factor of $\chi(p, q, \beta)$, then $p^{\prime}$ divides $p$. 
Proof. From the hypothesis there exists a closed invariant equivalence relation $R$ on $\chi(p, q, \beta)$ such that $\chi(p, q, \beta) / R$ is the cyclic flow on $p^{\prime}$ points. Define $R_{0}$ on $X^{D}$ by $(x, y) \in R_{0}$ if and only if $((x, 0),(y, 0)) \in R$, and check that $R_{0}$ is a closed invariant equivalence relation for $\left(X^{D}, q \beta\right)$. Clearly $X^{D} / R_{0}$ is finite and therefore

$$
R_{0}=X^{D} \times X^{D}
$$

because $\left(X^{D}, q \beta\right)$ is totally minimal. It follows that the cyclic flow on $p^{\prime}$ points is a factor of the cyclic flow on $p$ points.

THEOREM 3.4. If $(X, T)$ has TSMJ $\{$ PTMSJ $\}$ and $\alpha_{\pi}(d) \neq 0\left\{\alpha_{\pi}(d)>0\right\}$ for all $d \in D$, then the isomorphism classes of the minimal subsets of $\left(X^{D}, U(\pi, \alpha)\right)$ are in one-toone correspondence with the (positive) common divisors of $\rho$, the order of $\pi$, and $\delta$, the greatest common divisor of $\left\{\alpha_{\pi}(d): d \in D\right\}$. Furthermore, if $\sigma$ is a common divisor of $\rho$ and $\delta$, then $\chi(\rho / \sigma, 1,(1 / \sigma) \lambda)$ is a representative of the isomorphism class determined by $\sigma$ where $\lambda$ is the canonical injection determined by $\alpha_{\pi}$.

Proof. By remark 3.2 a minimal subset $M$ of $\left(X^{D}, U\right)$ has the form

$$
M=\bigcup_{i=0}^{\rho} U^{i} M_{0}
$$

where $M_{0}$ is minimal for $\alpha_{\pi}$. Let $k$ be the smallest positive integer such that $U^{k} M_{0}=M_{0}$. Clearly $k$ divides $\rho$ and $M_{0}=\prod_{j \in \Lambda} \Gamma\left(\beta_{j}\right)$. Set $\sigma=\rho / k$. Since $U^{k \sigma}=\alpha_{\pi}$, $U^{k} \mid M_{0}$ is an automorphism of $M_{0}$. Using the canonical isomorphism between $\left(X^{\Lambda}, \lambda\right)$ and $M_{0}$, and proposition 2.4 , we see that

$$
U^{k}\left|M_{0}=\gamma\right| M_{0}
$$

for some $\gamma \in \mathbb{Z}^{D}$ such that $\gamma$ is constant on each $D_{j}$. Thus $\alpha_{\pi}=\sigma \gamma, \sigma$ divides $\alpha_{\pi}(d)$ for all $d \in D$,

$$
U^{\kappa}\left|M_{0}=(1 / \sigma) \alpha_{\pi}\right| M_{0},
$$

and there exists a canonical isomorphism from $\chi(\rho / \sigma, 1,(1 / \sigma) \lambda)$. From proposition 3.3 it follows that different common divisors of $\rho$ and $\delta$ determine distinct isomorphism classes of the form $\chi(\rho / \sigma, 1,(1 / \sigma) \lambda)$. The remainder of the proof is devoted to showing that if $\sigma$ is a common divisor of $\rho$ and $\delta$, then for $k=\rho / \sigma$ there exists an $\alpha_{\pi}$-minimal set $M_{0}$ such that $U^{m} M_{0}=M_{0}$ if and only if $k$ divides $m$.

Let $\left\{D_{j}\right\}_{j \in \Lambda}$ be the canonical partition determined by $\alpha_{\pi}$. note that $\pi D_{j}=D_{j}$. Set $\pi_{j}=\pi \mid D_{j}$ and $\alpha_{j}=\alpha \mid D_{j}$. Then

$$
U(\pi, \alpha)=\prod_{j \in \Lambda} U\left(\pi_{j}, \alpha_{j}\right)
$$

The minimal set $M_{0}$ must have the form $\prod_{j \in \Lambda} \Gamma\left(\beta_{j}\right), \beta_{j} \in \mathbb{Z}^{D_{i j}}$. Hence $U^{k} M_{0}=M_{0}$ if and only if

$$
U_{j}^{k} \Gamma\left(\beta_{j}\right)=\Gamma\left(\beta_{j}\right)
$$

for all $j$ in $\Lambda$, where $U_{j}$ denotes $U\left(\pi_{j}, \alpha_{j}\right)$. Fix $j$ and let $d, d^{\prime} \in D_{j}$. Then $\alpha_{\pi}(d)=\alpha_{\pi}\left(d^{\prime}\right)$ which can be written

$$
\frac{1}{l(d)} \sum_{i=1}^{l(d)} \alpha\left(\pi^{-i}(d)\right)=\frac{1}{l\left(d^{\prime}\right)} \sum_{i=1}^{l\left(d^{\prime}\right)} \alpha\left(\pi^{-i}\left(d^{\prime}\right)\right)
$$


The version of $\alpha_{\pi}(d)=\alpha_{\pi}\left(d^{\prime}\right), d, d^{\prime} \in D_{i}$, given above says the average use of $T$ over a $\pi$ orbit is constant on $D_{j}$. In the light of equations (1) and (2) we study

$$
U^{k} \Gamma(\beta)=\Gamma(\beta)
$$

for arbitrary $k$ under the assumption that (2) holds for all $d, d^{\prime} \in D$.

It is a straightforward calculation to show that $U^{k} \Gamma(\beta)=\Gamma\left(\beta^{\prime}\right)$ where

$$
\beta^{\prime}(d)=\beta\left(\pi^{-k}(d)\right)+\sum_{i=1}^{k} \alpha\left(\pi^{-i}(d)\right) \text {. }
$$

Since $\Gamma(\beta)=\Gamma\left(\beta^{\prime}\right)$ if and only if there exists an integer $n$ such that $\beta^{\prime}=\beta+n \overline{1}$, $U^{k} \Gamma(\beta)=\Gamma(\beta)$ if and only if thre exists $n \in \mathbb{Z}$ such that

or

$$
\beta\left(\pi^{-k}(d)\right)+\sum_{i=1}^{k} \alpha\left(\pi^{-i}(d)\right)=\beta(d)+n
$$

$$
\beta(d)-\beta\left(\pi^{-k}(d)\right)=\sum_{i=1}^{k} \alpha\left(\pi^{-1}(d)\right)-n
$$

for all $d \in D$. If we fix $d$ and sum the above equation over the $\pi$ orbit of $d$, we find that

$$
n=k \frac{1}{l(d)} \sum_{i=1}^{l(d)} \alpha\left(\pi^{-i}(d)\right)
$$

which is independent of $d$ by the assumption that (2) holds. Therefore, $U^{k} \Gamma(\beta)=$ $\Gamma(\beta)$ if and only if whenever $D^{\prime}=\left\{\pi^{i}(d): 1 \leq i \leq l(d)\right\}$ for some $d \in D$

$$
U\left(\pi\left|D^{\prime}, \alpha\right| D^{\prime}\right)^{k} \Gamma\left(\beta \mid D^{\prime}\right)=\Gamma\left(\beta \mid D^{\prime}\right) \text {. }
$$

In other words, for a given $k$, to solve $U^{k}(\Gamma(\beta))=\Gamma(\beta)$ for $\beta$, it suffices to solve this problem for each cycle of $\pi$.

Proposition 3.5. Let $\pi=(1, \ldots, l)$ be a cycle, let $k$ be a positive integer, and let $\alpha \in \mathbb{Z}^{\prime}$ satisfying $\alpha_{\pi}(i) \neq 0\left\{\alpha_{\pi}(i)>0\right\}$ for $1 \leq i \leq l$. Set $U=U(\pi, \alpha)$ on $X^{\prime}$. then $U^{k}(\Gamma(\beta))=\Gamma(\beta)$ has a solution if and only if $l$ divides $k \sum_{i=1}^{l} \alpha(i)$. Moreover, when $l$ divides $k \sum_{i=1}^{l} \alpha(i)$, there exists $\beta \in \mathbb{Z}^{l}$ such that $U^{m}(\Gamma(\beta))=\Gamma(\beta)$ if and only if $(k, l)$ divides $m$.

Before proving this proposition we show how it can be used to complete the proof of theorem 3.4. As before, let $k=\rho / \sigma$ where $\sigma$ is a common divisor of $\rho$ and $\delta$. So, given $d \in D$, there exists $r \in \mathbb{Z}$ such that

$$
\sigma r=\alpha_{\pi}(d)=\frac{\rho}{l(d)} \sum_{i=1}^{l(d)} \alpha\left(\pi^{-i}(d)\right),
$$

because $\delta$ divides every $\alpha_{\pi}(d)$. Since the above equation can be written

$$
l(d) r=k \sum_{i=1}^{l(d)} \alpha\left(\pi^{-i}(d)\right) \text {, }
$$

the second part of the proposition can be applied to each cycle of $\pi$. Thus for each $j \in \Lambda$ there exists $\boldsymbol{\beta}_{j}$ in $\mathbb{Z}^{D_{i}}$ such that

$$
U\left(\pi_{j}, \alpha_{j}\right)^{k} \Gamma\left(\beta_{j}\right)=\Gamma\left(\beta_{j}\right)
$$


and hence $U^{k} M_{0}=M_{0}$. Moreover, $U^{m} M_{0}=M_{0}$ implies $(k, l(d))$ divides $m$ for all $d \in D$.

Suppose $m$ is the smallest positive integer such that $U^{m} M_{0}=M_{0}$. Then $m \mid k$ and $(m, l(d)) \leq(k, l(d))$ for all $d$. From $(k, l(d)) \mid m$, we get $(k, l(d)) \leq(m, l(d))$ and $(k, l(d))=(m, l(d))$ for all $d$. Since every prime power that divides $k$ also divides some $l(d), k=m$ and the proof of theorem 3.4 is complete.

Proof of proposition 3.5. Set

$$
n(k)=\frac{k}{l} \sum_{i=1}^{l} \alpha(i) .
$$

In this context solving $U^{k}(\Gamma(\beta))=\Gamma(\beta)$ for $\beta$ is equivalent to showing that $n(k) \in \mathbb{Z}$ and that the linear system

$$
\beta(j)-\beta\left(\pi^{-k}(j)\right)=\sum_{i=1}^{k} \alpha\left(\pi^{-i}(j)\right)-n(k)=f(j)
$$

has an integral solution. (Here $D=\{1, \ldots, l\}$.) In particular, $l$ dividing $k \sum_{i=1}^{l} \alpha(i)$ is an obvious necessary condition for the existence of a solution. To see that it is also sufficient first notice that the value of $\beta$ at $j$ will determine $\beta$ at every point of the $\pi^{k}$-orbit of $j$. This determination of $\beta$ will be consistent if and only if $f$ sums to 0 over every $\pi^{k}$-orbit. It is easy to check that for any positive integer $k, f$ sums to zero over every $\pi^{k}$ orbit. When $l$ divides $k \sum_{i=1}^{l} \alpha(i), f$ is integral valued. To determine $\beta$ we arbitrarily pick an integral value for $\beta$ at one point of each $\pi^{k}$ orbit.

For the second part we can assume without loss of generality that $k=(k, l)$. Suppose $k^{\prime} \mid k, k^{\prime} \neq k$. Because each $\pi^{k^{\prime}}$ orbit is a union of at least two different $\pi^{k}$ orbits, we can specify one integer value for each $\pi^{k}$ orbit so that the resulting $\beta$ is not a solution of $\beta$ for $k^{\prime}$. Clearly, this can be done simultaneously for all such $k^{\prime}$.

\section{The structure of factors for $\chi(p, q, \alpha)$ minimal sets}

Throughout this section it will be assumed that $(X, T)$ has TMSJ $\{$ PTMSJ $\}, \alpha \in \mathbb{Z}^{D}$ with $\alpha(d) \neq 0\{\alpha(d)>0\}$ for all $d \in D$, and $\left(X^{D}, \alpha\right)$ is minimal. It follows that $\chi(p, q, \alpha)$ is minimal for all positive integers $p$ and $q$. We can and will assume that the greatest common divisor of $\{\alpha(d): d \in D\}$ is 1 .

Before studying the factors of $\chi(p, q, \alpha)$, we show that the isomorphism class of $\chi(p, q, \alpha)$ is uniquely determined by $p$ and the range of $q \alpha$. Let $\alpha^{\prime} \in \mathbb{Z}^{D}$ satisfy the same conditions as $\alpha$ and ask: when are $\chi(p, q, \alpha)$ and $\chi\left(p^{\prime}, q^{\prime}, \alpha^{\prime}\right)$ isomorphic?

Proposition 4.1. The minimal sets $\chi(p, q, \alpha)$ and $\chi\left(p^{\prime}, q^{\prime}, \alpha^{\prime}\right)$ are isomorphic if and only if $p=p^{\prime}$ and there exists a bijection $\eta: D \rightarrow D^{\prime}$ such that

$$
q \alpha(d)=q^{\prime} \alpha^{\prime}(\eta(d))
$$

for all $d \in D$.

Proof. Assume $\chi(p, q, \alpha)$ and $\chi\left(p^{\prime}, q^{\prime}, \alpha^{\prime}\right)$ are isomorphic. It follows from proposition 3.3 that $p=p^{\prime}$. It is also clear that $\left(X^{D}, q \alpha\right)$ and $\left(X^{D^{\prime}}, q^{\prime} \alpha^{\prime}\right)$ are isomorphic. In particular, $\left(X, q^{\prime} \alpha^{\prime}\left(d^{\prime}\right)\right)$ is a factor of $\left(X^{D}, q \alpha\right)$ for each $d^{\prime} \in D$ and $\left(X^{D} \times X, q \alpha \times\right.$ $q^{\prime} \alpha^{\prime}\left(d^{\prime}\right)$ ) is not minimal. Therefore, there exists a unique $d \in D$ such that $q \alpha(d)=$ $q^{\prime} \alpha^{\prime}\left(d^{\prime}\right)$. The rest of the proof is routine. 
Let $(Y, S)$ be a factor of $\chi(p, q, \alpha)$ and let $R$ be the corresponding invariant closed equivalence relation on $\chi(p, q, \alpha)$. We will assume that $R$ does not equal the diagonal. Since, using proposition 2.4 , it is easy to show that every endomorphism of $\chi(p, q, \alpha)$ is an automorphism, $(Y, S)$ cannot be isomorphic to $\chi(p, q, \alpha)$. As in the proof of proposition 3.3 let $R_{0}$ denote the invariant closed equivalence relation on $\left(X^{D}, q \alpha\right)$ given by $(x, y) \in R_{0}$ if and only if $((x, 0),(y, 0)) \in R$. Note the following:

Remark 4.2. The factor $(Y, S)$ is finite if and only if $R_{0}=X^{D} \times X^{D}$.

Let $E$ be any subset of $D$ and define the relation $R(E)$ on $\chi(p, q, \alpha)$ by

$$
R(E)=\{((x, i),(y, j)): i=j \text { and } x(d)=y(d) \text { for all } d \in E\}
$$

Remark 4.3. $R(E)$ is an invariant closed equivalence relation on $\chi(p, q, \alpha)$. Moreover, $\chi(p, q, \alpha) / R(E)$ is isomorphic to $\chi(p, q, \alpha \mid E)$ when $E \neq \varnothing$, and the cyclic flow on $p$ points when $E=\varnothing$.

When $R=R(E)$ for some subset $E \subset D, E \neq D$, we will say $\chi(p, q, \alpha)$ is a Cartesian extension of $(Y, S)$.

Proposition 4.4. If $R_{0}$ is not contained in the proximal relation of $\left(X^{D}, \alpha\right)$, then there exists a smallest subset $E \subset D$ such that $R(E) \subset R$ and $E \neq D$.

Proof. Let $(x, y)$ be an element of $R_{0}$ with $x$ and $y$ not proximal. Then the orbit closure of $(x, y)$ in $\left(X^{D} \times X^{D}, \alpha \times \alpha\right)$ contains a minimal set $M$ different from the diagonal. As in the proof of proposition 2.4, there exists $\beta \in \mathbb{Z}^{D}$ such that

$$
M=\left\{(x, \beta x): x \in X^{D}\right\}
$$

Clearly $\beta \neq 0$. Let $E=\{d: \beta(d)=0\} \neq D$. Set $E^{\prime}=D \backslash E$ and $\beta^{\prime}=\beta \mid E^{\prime}$. Hence $\left(X^{E^{\prime}}, \beta^{\prime}\right)$ is weak mixing and we can find $u \in X^{E^{\prime}}$ with a dense $\beta^{\prime}$ orbit. Consequently, for $w \in X^{E}$,

$$
\left((u, w),\left(k \beta^{\prime} u, w\right)\right) \in R_{0} \quad \text { for all } k \in \mathbb{Z}
$$

which implies $R(E) \subset R$. (This part of the argument comes from [7, proposition 3.3].) Now set

$$
\mathscr{E}=\{E \subset D: R(E) \subset R\} .
$$

Note that $E_{1} \subset E_{2}$ implies $R\left(E_{1}\right) \supset R\left(E_{2}\right)$ and that $E_{1}, E_{2} \in \mathscr{E}$ implies $E_{1} \cap E_{2} \in \mathscr{E}$. Set

$$
F=\bigcap\{E: E \in \mathscr{E}\} .
$$

Since $\mathrm{Cl}(\bigcup\{R(E): E \in \mathscr{E}\}) \subset R$, it suffices to show that

$$
R(F) \subset \mathrm{Cl}(\bigcup\{R(E): E \in \mathscr{E}\}) \text {. }
$$

Let $((x, i),(y, i) \in R(F)$ and let $W$ be a neighbourhood of $(x, y)$. There exists a finite set $J \subset D$ such that if $y^{\prime}(d)=y(d)$ for all $d \in J$, then $\left(x, y^{\prime}\right) \in W$. Set

$$
J_{1}=J \cap F \text { and } J_{2}=J \backslash J_{1} \text {. }
$$

There exists $D^{\prime} \in \mathscr{E}$ such that $D^{\prime} \cap J_{2}=\varnothing$. Now define $y^{\prime}$ in $X^{D}$ by $y^{\prime}(d)=y(d)$ for all $d \in J$ and $y^{\prime}(d)=x(d)$ for all $d \notin J$. Then $\left(x, y^{\prime}\right) \in W \cap R\left(D^{\prime}\right)_{0}$ and it follows that

$$
((x, i),(y, i)) \in \mathrm{Cl}(\bigcup\{R(E): E \in \mathscr{E}\}) .
$$


Proposition 4.5. If $R_{0}$ equals the diagonal, then $\chi(p, q, \alpha)$ is a finite group extension of $(Y, S)$ and there exists a common divisor $\sigma$ of $p$ and $q$ such that $(Y, S)$ is isomorphic to $\chi(p / \sigma, q / \sigma, \alpha)$.

Proof. Let $i$ be the smallest positive integer for which $((x, 0),(y, i)) \in R$ for some $x$ and $y$ in $X^{D}$. Because $\left(X^{D}, q \alpha\right)$ is minimal, for every $x$ there exists $y$ such that $((x, 0),(y, i)) \in R$ and $y$ is uniquely determined by $x$ since $R_{0}=\Delta$. Clearly the function $x \rightarrow y$ is an automorphism of $\left(X^{D}, q \alpha\right)$. Hence there exists $\gamma \in \mathbb{Z}^{D}$ such that $((x, 0),(\gamma x, i)) \in R$ for all $x$. Define $\theta: \chi(p, q, \alpha) \rightarrow \chi(p, q, \alpha)$ by

$$
\theta(x, j)= \begin{cases}(\gamma x, j+i) & \text { if } j+i<p \\ ((q \alpha+\gamma) x, j+i-p) & \text { if } j+i \geq p\end{cases}
$$

and check that $\theta$ is an automorphism of $\chi(p, q, \alpha)$. It follows that $((x, j), \theta(x, j)) \in R$ for all $(x, j)$.

From the choice of $i$ it follows that $i$ divides $p$ and then $\theta^{p / i}=$ identity because $R_{0}=\Delta$. Thus

$$
q \alpha+(p / i) \gamma=0
$$

It also follows from the choice of $i$ that the $\theta$ orbits are precisely the equivalence classes for $R$. Thus $\chi(p, q, \alpha)$ is a finite group extension of $(Y, S)$.

Now set $\sigma=p / i$. Then $q \alpha+\sigma \gamma=0$ implies $\sigma \mid q$ and $\gamma=-(q / \sigma) \alpha$. Set $p^{\prime}=p / \sigma$ and $q^{\prime}=q / \sigma$, and write $j=m p^{\prime}+r$ with $0 \leq r<p^{\prime}$. Define $\Psi: \chi(p, q, \alpha) \rightarrow \chi\left(p^{\prime}, q^{\prime}, \alpha\right)$ by

$$
\Psi(x, j)=\left(m q^{\prime} \alpha x, r\right) .
$$

Finally, to complete the proof one checks that $\Psi(x, j)=\Psi\left(x^{\prime}, j^{\prime}\right), j<j^{\prime}$, if and only if $\theta^{k}(x, j)=\left(x^{\prime}, j^{\prime}\right)$ where $k=\left(j^{\prime}-j\right) / p^{\prime}$.

Since $\theta$ constructed as above is an automorphism for any $i, 0 \leq i<p$, and any $\gamma \in \mathbb{Z}^{D}$, the previous paragraph proves the following corollary:

Corolllary 4.6. If $\sigma$ is a common divisor of $p$ and $q$, then $\chi(p, q, \alpha)$ is a finite group extension of $\chi(p / \sigma, q / \sigma, \alpha)$.

COROLlARY 4.7. The relation $R_{0}$ equals the diagonal if and only if $\chi(p, q, \alpha)$ is a finite group extension of $(Y, S)$.

Proof. Let $\theta$ be an automorphism of $\chi(p, q, \alpha)$ of finite order and suppose that $\theta(x, 0)=(y, 0)$. It follows that $\theta(x, j)=(\gamma x, j)$ for some $\gamma \in \mathbb{Z}^{D}$ and then $\gamma=0$.

Actually slightly more is true about the automorphisms of $\chi(p, q, \alpha)$. Every automorphism is of the form $\theta$ constructed in the proof of proposition 4.5. The torsion subgroup of the group of automorphisms is the finite cyclic group of order $\delta=(p, q)$ generated by the $\theta$ constructed with $i=p / \delta$ and $\gamma=-(q / \delta) \alpha$.

THEOREM 4.8. If $(Y, S)$ is a factor of a minimal flow $\chi(p, q, \alpha)$ constructed from a flow having TMSJ $\{\mathrm{PTMSJ}\}$, then exactly one of the following holds:

(I) $\chi(p, q, \alpha)$ is a Cartesian extension of $(Y, S)$;

(II) $\chi(p, q, \alpha)$ is a finite group extension of $(Y, S)$;

(III) $\chi(p, q, \alpha)$ is a proper proximal extension of a distal extension of $(Y, S)$. 
(The distal extension need not be proper.)

(IV) there exists a subset $E$ of $D$ such that $\chi(p, q, \alpha \mid E)$ is a proper extension of (Y,S) satisfying (II) or (III).

Proof. Observe that the following conditions on $R$ are mutually exclusive:

(i) $R=R(E)$ for some subset $E$ of $D$ with $E \neq D$;

(ii) $R_{0}=\Delta$;

(iii) $\Delta \neq R_{0} \subset P$;

(iv) $R \supset R(E)$ for some subset $E$ of $D$ with $E \neq D$ and $R \neq R(F)$ for all $F \subset D$.

If none of (i), (ii), and (iii) holds, then using proposition 4.4 it follows that (iv) holds. We also know that (i) and (ii) are equivalent to (I) and (II), respectively. To complete the proof it suffices to show that (iii) and (iv) are equivalent to (III) and (IV), respectively.

If $\Delta \neq R_{0} \subset P$, then

$$
\tilde{R}=\left\{((x, i),(y, j)): i=j \text { and }(x, y) \in R_{0}\right\}
$$

is an invariant closed equivalence relation on $\chi(p, q, \alpha)$ such that $\Delta \neq \tilde{R} \subset P$. It is routine to check that the homomorphism from $\chi(p, q, \alpha) / \tilde{R}$ to $(Y, S)$ is distal.

Now suppose (III) holds. Then there exists an invariant closed equivalence relation

$$
\Delta \neq \tilde{R} \subset R \cap P \text {. }
$$

Obviously, $\Delta \neq \tilde{R}_{0} \subset P$. If $R_{0}$ is not contained in $P$, then $R(E) \subset R$ for some $E \neq D$. Without loss of generality, $E=D \backslash\left\{d_{0}\right\}$. Fix $w \in X$ and for $u \in X$ let $x_{u} \in X^{D}$ be defined by $x_{u}(d)$ equals $w$ or $u$ according to whether $d \neq d_{0}$ or $d=d_{0}$. Then

$$
C=\left\{(u, v):\left(x_{u}, x_{v}\right) \in \tilde{R}_{0}\right\}
$$

is closed in $X^{2}$. Set $W=X^{2} \mid C$. If $W=\varnothing$, then $\tilde{R}_{0}$ contains points not in $P$. If $W \neq \varnothing$, then

$$
(R \backslash \tilde{R}) \cap P \neq \varnothing
$$

because the proximal relation is dense in $X^{2}$. This contradicts the distal part of (III).

Next assume (iv) holds. Clearly, $R_{0} \not \subset P$ and, by proposition 4.4 , there exists a smallest set $E$ such that $R(E) \subset R$. Then $(Y, S)$ is a factor of $\chi(p, q, \alpha \mid E)$ and determines an invariant closed equivalence relation $\tilde{R}$ on $\chi(p, q, \alpha \mid E)$. Because $R(E) \neq R, \tilde{R} \neq \Delta$ and satisfies one of the conditions (i), (ii), (iii) or (iv). By the choice of $E$, (i) and (iv) are impossible.

Finally, assume (IV). Obviously, there exists $E \neq D$ such that $R(E) \subset R$ and $R(E) \neq R$. Also the induced invariant closed equivalence relation $\tilde{R}$ on $\chi(p, q, \alpha \mid E)$ satisfies (ii) or (iii). If $R=R(F)$ for some $F \subset D$, then it follows that $F \subset E$ and $\tilde{R}=R(F)$ where $F$ is viewed as a subset of $E$. This contradicts the fact that $\tilde{R}$ satisfies exactly one of the conditions (i) to (iv). Hence, $R$ satisfies (iv) and the proof is finished.

COROllary 4.9. If $(X, T)$ is a POD flow and $(Y, S)$ is a factor of the minimal flow $\chi(p, q, \alpha)$, then exactly one of the following holds:

(a) $\chi(p, q, \alpha)$ is a Cartesian extension of $(Y, S)$; 
(b) $\chi(p, q, \alpha)$ is a finite extension of $(Y, S)$;

(c) there exists a subset $E$ of $D$ with $E \neq D$ such that $\chi(p, q, \alpha \mid E)$ is a finite group extension of $(Y, S)$.

Proof. It suffices to show that (iii) in the previous proof is impossible when $(X, T)$ is POD. Suppose $(x, y) \in R_{0}$ with $x \neq y$. Because $(X, T)$ is POD, lemma 3.2 from [7] applies and there exists $\gamma \in \mathbb{Z}^{D}, \gamma \neq 0$, such that

$$
\left\{(x, \gamma x): x \in X^{D}\right\} \subset R_{0} \text {. }
$$

It follows that $R_{0}$ is not contained in the proximal relation when $R_{0} \neq \Delta$.

THEOREM 4.10. Suppose the minimal flows $\chi\left(p_{1}, q_{1}, \alpha_{1}\right)$ and $\chi\left(p_{2}, q_{2}, \alpha_{2}\right)$ are constructed from the same $(X, T)$ with TMSJ $\left\{\right.$ PTMSJ\} and the $\alpha_{i}$ satisfy the usual conditions. Then the following are equivalent:

(1) $\chi\left(p_{1}, q_{1}, \alpha_{1}\right)$ and $\chi\left(p_{2}, q_{2}, \alpha_{2}\right)$ are disjoint, i.e. $\chi\left(p_{1}, q_{1}, \alpha_{1}\right) \times \chi\left(p_{2}, q_{2}, \alpha_{2}\right)$ is minimal;

(2) $\chi\left(p_{1}, q_{1}, \alpha_{1}\right)$ and $\chi\left(p_{2}, q_{2}, \alpha_{2}\right)$ do not have a common non-trivial factor;

(3) $\left(p_{1}, p_{2}\right)=1$ and for all $d_{i} \in D_{i}, q_{1} \alpha_{1}\left(d_{1}\right) / p_{1} \neq q_{2} \alpha_{2}\left(d_{2}\right) / p_{2}$.

Proof. Since (1) implies (2) is well known, we begin with (2) implies (3). Because there exists a finite common factor containing $\left(p_{1}, p_{2}\right)$ points, $\left(p_{1}, p_{2}\right)=1$. Now, suppose there exist $d_{1} \in D_{1}$ and $d_{2} \in D_{2}$ such that

$$
q_{1} \alpha_{1}\left(d_{1}\right) / p_{1}=q_{2} \alpha_{2}\left(d_{2}\right) / p_{2} .
$$

Because $\left(p_{1}, p_{2}\right)=1, p_{1} \mid q_{1} \alpha_{1}\left(d_{1}\right)$ and $p_{2} \mid q_{2} \alpha_{2}\left(d_{2}\right)$. Set

$$
r=\frac{q_{1} \alpha_{1}\left(d_{1}\right)}{p_{1}}=\frac{q_{2} \alpha_{2}\left(d_{2}\right)}{p_{2}} \in \mathbb{Z}
$$

It follows that

$$
\left(X, T^{r}\right)=\chi(1, r, 1)=\chi\left(p_{i} / p_{i}, q_{i} \alpha_{i}\left(d_{i}\right) / p_{i}, 1\right),
$$

$i=1,2$, and is a common non-trivial factor because $\chi\left(p_{i}, q_{i}, \alpha_{i} \mid\left\{d_{i}\right\}\right)$ is a Cartesian factor of $\chi\left(p_{i}, q_{i}, \alpha_{i}\right), i=1,2$.

To complete the proof we show that (3) implies (1). Set $D=D_{1} \cup D_{2}$ and define $\alpha \in \mathbb{Z}^{D}$ by

$$
\alpha \mid D_{1}=p_{2} q_{1} \alpha_{1} \quad \text { and } \quad \alpha \mid D_{2}=p_{1} q_{2} \alpha_{2} .
$$

Because $\left(p_{1}, p_{2}\right)=1$, the disjointness is equivalent to the minimality of $\left(X^{D}, \alpha\right)$ which follows from proposition 2.3. (In fact, we have also shown that $\chi\left(p_{1}, q_{1}, \alpha_{1}\right) \times$ $\chi\left(p_{2}, q_{2}, \alpha_{2}\right)$ is isomorphic to $\chi\left(p_{1} p_{2}, 1, \alpha\right)$ where (3) holds.)

One of the striking theorems in Rudolph's paper [9, theorem 3.1] says that if $U(\pi, \alpha)$ and $U\left(\pi^{\prime}, \alpha^{\prime}\right)$ are ergodic, measure theoretically isomorphic, and the underlying transformation has MSJ, then the isomorphism is the composition of a $\beta \in \mathbb{Z}^{D}$ followed by a rearranging of the coordinates. The author does not know whether or not such a result holds for TMSJ. In fact, it is unknown whether or not $\left(X^{2}, \overline{1}\right)$ and $\left(X^{3}, \overline{1}\right)$ can be isomorphic when $(X, T)$ is a POD flow. 
We conclude this section with some necessary conditions for $U(\pi, \alpha)$ to be isomorphic to $U\left(\pi^{\prime}, \alpha^{\prime}\right)$ in the TMSJ setting. Given $\pi$ and $\alpha$, set

$$
\operatorname{Ave}(\pi, \alpha)=\left\{\frac{1}{l(d)} \sum_{i=1}^{l(d)} \alpha\left(\pi^{i}(d)\right): d \in D\right\} \text {. }
$$

In other words, for $d \in D$, we calculate the average use of $T$ over the $\pi$ orbit of $d$ and let Ave $(\pi, \alpha)$ be the set of all rational numbers obtained this way as $d$ runs through $D$.

THEOREM 4.11 Let $\pi$ and $\pi^{\prime}$ have finite orders $\rho$ and $\rho^{\prime}$ respectively, and suppose $U(\pi, \alpha)$ and $U\left(\pi^{\prime}, \alpha^{\prime}\right)$ are weak mixing. If $\left(X^{D^{\prime}}, U\left(\pi^{\prime}, \alpha^{\prime}\right)\right)$ is a factor of $\left(X^{D}, U(\pi, \alpha)\right)$, then $\rho^{\prime}$ divides $\rho$ and Ave $\left(\pi^{\prime}, \alpha^{\prime}\right) \subset$ Ave $(\pi, \alpha)$.

Proof. If $\left(X^{D^{\prime}}, U\left(\pi^{\prime}, \alpha^{\prime}\right)\right)$ is a factor of $\left(X^{D}, U(\pi, \alpha)\right)$, then $\chi\left(\rho^{\prime}, 1, \lambda^{\prime}\right)$ is a factor of $\chi(\rho, 1, \lambda)$, where as usual $\lambda$ and $\lambda^{\prime}$ denote the canonical injections determined by $\alpha_{\pi}$ and $\alpha_{\pi^{\prime}}^{\prime}$. Clearly, $\rho^{\prime}$ divides $\rho$, and by theorem $4.8, \chi\left(\rho^{\prime}, 1, \lambda^{\prime}\right)$ falls into one of four categories. It is easy to verify the following:

(i) If $\chi(\rho, 1, \lambda)$ is a Cartesian extension of $\chi\left(\rho^{\prime}, 1, \lambda^{\prime}\right)$, then

$$
\operatorname{Ave}\left(\pi^{\prime}, \alpha^{\prime}\right) \subset \operatorname{Ave}(\pi, \alpha)
$$

(ii) If $\chi(\rho, 1, \lambda)$ is a finite group extension of $\chi\left(\rho^{\prime}, 1, \lambda^{\prime}\right)$, then

$$
\text { Ave }\left(\pi^{\prime}, \alpha^{\prime}\right)=\operatorname{Ave}(\pi, \alpha)
$$

These facts combined with the next proposition complete the proof.

Proposition 4.12. If $(Y, S)$ is a factor of the minimal flow $\chi(p, q, \alpha)$, then the following are incompatible:

(a) $\chi(p, q, \alpha)$ is a proper proximal extension of a distal extension of $(Y, S)$;

(b) $(Y, S)$ is isomorphic to some $\chi\left(p^{\prime}, q^{\prime}, \alpha^{\prime}\right)$.

Proof. Let $\theta: \chi(p, q, \alpha) \rightarrow \chi\left(p^{\prime}, q^{\prime}, \alpha^{\prime}\right)$ be a homomorphism such that the equivalence relation $R$ determined by $\theta$ satisfies $\Delta \neq R_{0} \subset P$. In the light of the proof of theorem 4.8, this amounts to (a) and (b) holding simultaneously. Clearly, $p^{\prime} \mid p$ and $\left(X^{D^{\prime}},\left(p / p^{\prime}\right) q^{\prime} \alpha^{\prime}\right)$ is the factor of $\left(X^{D}, q \alpha\right)$ determined by $R_{0}$. Using the idea of the proof of proposition 4.1, we can show that, without loss of generality, $D^{\prime} \subset D$ and

$$
p^{\prime} q \alpha\left(d^{\prime}\right)=p q^{\prime} \alpha^{\prime}\left(d^{\prime}\right) \quad \text { for all } d^{\prime} \in D^{\prime} \text {. }
$$

If $D=D^{\prime}$, then $R_{0}$ determines an endomorphism of $\left(X^{D}, q \alpha\right)$. Since every endomorphism of $\left(X^{D}, q \alpha\right)$ is an automorphism (proposition 2.4), $R_{0}$ would equal the diagonal, contrary to our assumption. Therefore, we can select $d_{0} \in D \backslash D^{\prime}$.

Choose $x, y \in X^{D}$ such that $x(d)=y(d)$ for all $d \neq d_{0}$ and $x\left(d_{0}\right)=T y\left(d_{0}\right)$. Then $(x, y) \notin P$ and $(x, y) \notin R_{0}$. Notice that

$$
\left(X^{D^{\prime}} \times X, p q^{\prime} \alpha^{\prime} \times p^{\prime} q \alpha\left(d_{0}\right)\right)
$$

is minimal. Using $\theta$ also to denote the homomorphism of $\left(X^{D}, q \alpha\right)$ onto $\left(X^{D^{\prime}}, q^{\prime} \alpha^{\prime}\right)$ and letting

$$
\gamma=p q^{\prime} \alpha^{\prime} \times p^{\prime} q \alpha\left(d_{0}\right),
$$

there exists a sequence of positive integers $k_{i}$ such that

$$
k_{i} \gamma\left(\theta(x), x\left(d_{0}\right)\right) \rightarrow\left(\theta(y), x\left(d_{0}\right)\right) .
$$


By taking a subsequence, we can assume

$$
k_{i} p^{\prime} q \alpha x \rightarrow y^{\prime} \text {. }
$$

Then $\left(y, y^{\prime}\right) \in R_{0} \subset P$. It follows that $y^{\prime}\left(d_{0}\right)=x\left(d_{0}\right)$ which produces the contradiction

$$
\left(y\left(d_{0}\right), T y\left(d_{0}\right)\right)=\left(y\left(d_{0}\right), x\left(d_{0}\right)\right)=\left(y\left(d_{0}\right), y^{\prime}\left(d_{0}\right)\right) \quad \in P .
$$

5. $U(\pi, \alpha)$ minimal sets: infinite order case

Let $\pi$ be a compact permutation of the countable set $D$. Assume $\pi$ has infinite order. It follows that $D$ is infinite and $l(D)$ is an infinite subset of $\mathbb{Z}$. We will write

$$
l(D)=\left\{e_{0}<e_{1}<\cdots<e_{k}<\cdots\right\}
$$

Now define the sequence of integers $\left\{\rho_{k}\right\}$ inductively as follows:

$$
\rho_{0}=e_{0}
$$

$\rho_{k}=$ least common multiple of $\rho_{k-1}$ and $e_{i(k)}$ where $i(k)$ is the smallest positive integer such that the least common multiple of $\rho_{k-1}$ and $e_{i}$ does not equal $\rho_{k-1}$.

Note $i(k) \geq k, \rho_{k-1}<\rho_{k}$, and $\rho_{k-1} \mid \rho_{k}$. Set

$$
D_{k}=\left\{d \in D: l(d) \mid \rho_{k}\right\}, \quad \pi_{k}=\pi \mid D_{k} \quad \text { and } \quad \alpha_{k}=\alpha \mid D_{k} .
$$

Then $\pi_{k}$ is a permutation of $D_{k}$ of order $\rho_{k}$ and the projection of $X^{D}$ onto $X^{D_{k}}$ is a homomorphism of $\left(X^{D}, U(\pi, \alpha)\right)$ onto $\left(X^{D_{k}}, U\left(\pi_{k}, \alpha_{k}\right)\right)$. Moreover, projections provide homomorphisms of $\left(X^{D_{k}}, U\left(\pi_{k}, \alpha_{k}\right)\right)$ onto $\left(X^{D_{k-1}}, U\left(\pi_{k-1}, \alpha_{k-1}\right)\right)$ and $\left(X^{D}, U(\pi, \alpha)\right)$ is isomorphic to the inverse limit of $\left(X^{D_{k}}, U\left(\pi_{k}, \alpha_{k}\right)\right)$ as $k$ goes to infinity.

It will again be assumed that $(X, T)$ has TMSJ $\{$ PTMSJ $\}$ and that $\left(X^{D}, U(\pi, \alpha)\right)$ is weak mixing.

Remark 5.1. The flow $\left(X^{D}, U(\pi, \alpha)\right)$ is weak mixing if and only if

for all $d \in D$.

$$
\sum_{i=1}^{l(d)} \alpha\left(\pi^{i}(d)\right) \neq 0
$$

Let $M$ be a minimal subset of $U(\pi, \alpha)$ and let $M_{k}$ be its image in $X^{D_{k}}$. Clearly $M$ is isomorphic to the inverse limit of the $M_{k}$ 's and each $M_{k}$ is a $U\left(\pi_{k}, \alpha_{k}\right)$ minimal set. Since $\pi_{k}$ has finite order $\rho_{k}$, for each $k$ there exists a canonical $\chi\left(\rho_{k}, 1, \lambda_{k}\right)$ which is a finite group extension of $M_{k}$. The idea is to study $M$ via the sequence of minimal sets $\chi\left(\rho_{k}, 1, \lambda_{k}\right)$.

Let $\beta \in \mathbb{Z}^{D}$ such that $\left(X^{D}, \beta\right)$ is minimal. Given a non-empty subset $E$ of $D$, there is a natural homomorphism

namely

$$
\theta_{E}: \chi(p, q, \beta) \rightarrow \chi(p, q, \beta \mid E),
$$

$$
\theta_{E}(x, i)=(x \mid E, i)
$$

If $\sigma$ divides $p$ and $q$, then there is a natural homomorphism

$$
\Psi_{\sigma}: \chi(p, q, \beta) \rightarrow \chi(p / \sigma, q / \sigma, \beta)
$$

as defined in the proof of proposition 4.5. Homomorphisms of the form $\Psi_{\sigma} \circ \theta_{E}$ 
where $E$ is a non-empty subset of $D$ and $\sigma \neq 1$ will be called increasing homomorphisms.

Remark 5.2. Given a non-empty subset $E$ of $D$ and $\beta^{\prime} \in \mathbb{Z}^{E}$, there exists an increasing homomorphism $\varphi: \chi(p, q, \beta) \rightarrow \chi\left(p^{\prime}, q^{\prime}, \beta^{\prime}\right)$ if and only if $p^{\prime}$ divides $p$ and $\left(p / p^{\prime}\right) q^{\prime} \beta^{\prime}=q(\beta \mid E)$.

By an increasing inverse limit system for $(X, T)$, we shall mean a sequence

$$
\left(\chi\left(p_{k}, q_{k}, \beta_{k}\right), \varphi_{k}\right), \quad k=0,1, \ldots,
$$

of minimal flows $\chi\left(p_{k}, q_{k}, \beta_{k}\right)$ constructed from $(X, T)$ and increasing homomorphisms

$$
\varphi_{k}: \chi\left(p_{k+1}, q_{k+1}, \alpha_{k+1}\right) \rightarrow \chi\left(p_{k}, q_{k}, \alpha_{k}\right)
$$

Of course, the inverse limit of an increasing inverse limit system is a minimal flow.

Next we want to construct a set $\Lambda$ such that the $\lambda_{k}$ 's in $\chi\left(\rho_{k}, 1, \lambda_{k}\right)$ are naturally defined on a sequence of subsets $\Lambda_{k}$ satisfying

$$
\Lambda_{k} \subset \Lambda_{k+1} \text { and } \Lambda=\bigcup_{k=0}^{\infty} \Lambda_{k}
$$

For $d \in D_{k}$, set

$$
\bar{\alpha}_{k}(d)=\frac{\rho_{k}}{l(d)} \sum_{i=1}^{l(d)} \alpha\left(\pi^{i}(d)\right)
$$

and note that

$$
\left(\rho_{k} / \rho_{k-1}\right) \bar{\alpha}_{k-1}=\bar{\alpha}_{k} \mid D_{k-1} .
$$

(In the notation of $\S 3, \bar{\alpha}_{k}$ would have been written cumbersomely as $\alpha_{k}$ with $\pi_{k}$ as a subscript.) Then $\bar{\alpha}_{k}(d)=\bar{\alpha}_{k}\left(d^{\prime}\right)$ for some $k$ such that $d, d^{\prime} \in D_{k}$ defines an equivalence relation on $D$. Let $\Lambda$ be the set of equivalence classes, let

$$
\Lambda_{k}=\left\{\xi \in \Lambda: \xi \cap D_{k} \neq \varnothing\right\},
$$

and let

$$
\lambda_{k}(\xi)=\bar{\alpha}_{k}(d) \quad \text { for } d \in \xi \cap D_{k} .
$$

Observe that for each $k$ there exists an increasing homomorphism

$$
\varphi_{k}: \chi\left(\rho_{k+1}, 1, \lambda_{k+1}\right) \rightarrow \chi\left(\rho_{k}, 1, \lambda_{k}\right)
$$

to produce an increasing inverse system.

Proposition 5.3. Every minimal subset of $\left(X^{D}, U(\pi, \alpha)\right)$ is a factor of the inverse limit of the increasing inverse system $\left(\chi\left(\rho_{k}, 1, \lambda_{k}\right), \varphi_{k}\right)$ for $(X, T)$. Moreover, the inverse limit of this system does occur as a minimal subset of $\left(X^{D}, U(\pi, \alpha)\right)$.

Proof. The first statement follows from an easy diagram chase. The proof of theorem 3.4 shows how to construct $M_{j}$ cycle-by-cycle using proposition 3.5 . We apply proposition 3.5 to each cycle in $D_{j}$ with $k=\left(\rho_{j}, l\right)=l$ the length of the cycle. This produces a minimal subset $M_{j}$ isomorphic to $\chi\left(\rho_{j}, 1, \lambda_{j}\right)$.

Observe that if $r$ is a positive integer, then every solution of the system $\left(L_{k}\right)$ is a solution of $\left(L_{r k}\right)$. Consequently, when we go to construct $M_{j+1}$ isomorphic to 
$\chi\left(\rho_{j+1}, 1, \lambda_{j+1}\right)$, everything we used for $M_{j}$ can be reused. In particular, $M_{j+1}$ will project onto $M_{j}$ which completes the proof.

Consider an increasing inverse system $\left(\chi\left(p_{k}, q_{k}, \beta_{k}\right), \varphi_{k}\right)$. Then $D_{k} \subset D_{k+1}$ for all $k$, $p_{k}<p_{k+1}, p_{k} \mid p_{k+1}$. Set

$$
D=\bigcup_{k=0}^{\infty} D_{k}, \quad p_{-1}=1, \quad \text { and } \quad a_{k}=p_{k+1} / p_{k} .
$$

Now let

$$
\Delta_{a}=\left\{\left(z_{0}, z_{1}, \ldots, z_{k}, \ldots\right): z_{i} \in \mathbb{Z} \text { and } 0 \leq z_{k} \leq a_{k}\right\}
$$

denote the group of $a$-adic integers associated with the sequence $a=$ $\left(a_{0}, a_{1}, \ldots, a_{k}, \ldots\right)$. Thus, $\Delta_{a}$ is a compact monthetic group with generator $\mathbf{1}=$ $(1,0, \ldots, 0, \ldots)$. For each non-negative integer

$$
\Gamma_{k}=\left\{z \in \triangle_{a}: z_{i}=0 \text { for } i \leq k\right\}
$$

is an open closed subgroup of $\Delta_{a}$.

Define $f: \Delta_{a} \rightarrow \mathbb{Z}^{D}$ by

$$
f(z)(d)= \begin{cases}q_{k} \beta_{k}(d) & \text { if } z \in \Gamma_{k}, \quad d \in D_{k} \backslash D_{k-1}, \\ 0 & \text { otherwise. }\end{cases}
$$

If we put the product topology on $\mathbb{Z}^{D}$ coming from the discrete topology on $\mathbb{Z}$, then $f$ is continuous and so is the natural action of $\mathbb{Z}^{D}$ on $X^{D}$. Consequently, we can define a flow

by

$$
\omega(a, f)=\left(X^{D} \times \triangle_{a}, F\right)
$$

$$
F(x, z)=(f(z+1) x, z+1) .
$$

For each positive integer $k$ we define an invariant closed equivalence relation $R_{k}$ on $X^{D} \times \triangle_{a}$ by $(x, z) \sim\left(x^{\prime}, z^{\prime}\right)$ if and only if $x(d)=x^{\prime}(d)$ for all $d \in D_{k}$ and $z-z^{\prime} \in \Gamma_{k}$. Clearly, $X^{D} \times \triangle_{a} / R_{k}$ is homeomorphic to $X^{D_{k}} \times\left\{0,1, \ldots, p_{k}\right\}$. The induced action $F_{k}$ is given by $F_{k}(x, i)=(y, j)$ where

(a) $j=i+1 \quad$ if $i+1<p_{k}$

$$
j=0 \quad \text { if } i+1=p_{k} \text {, and }
$$

(b) for $d \in D_{j} \backslash D_{j-1}, j \leq k$,

$$
\begin{aligned}
& y(d)=q_{j} \beta_{j}(d) x(d) \quad \text { if } p_{j} \mid(i+1) \\
& y(d)=x(d) \quad \text { otherwise. }
\end{aligned}
$$

Let $\hat{\varphi}_{k}$ denote the induced homomorphism of $\left(X^{D_{k+1}} \times\left\{0,1, \ldots, p_{k+1}\right\}, F_{k+1}\right)$ onto $\left(X^{D_{k}} \times\left\{0,1, \ldots, p_{k}\right\}, F_{k}\right)$.

We can define a map $\theta_{k}$ of $\chi\left(p_{k}, 1, \beta_{k}\right)$ to $\left(X^{D_{k}} \times\left\{0,1, \ldots, p_{k}\right\}, F_{k}\right)$ by

$$
\theta_{k}(x, i)=(y, i)
$$

and for $d \in D_{j} \mid D_{j-1}$

$$
y(d)=\delta q_{j} \beta_{j}(d) x(d),
$$

where $i=r+\delta p_{j}$ and $0 \leq r<p_{j}$. It is easy to check that $\theta_{k}$ is an isomorphism and

$$
\theta_{k} \circ \varphi_{k}=\hat{\varphi}_{k} \circ \theta_{k+1} \text {. }
$$


Consequently, the inverse system

$$
\left(X^{D_{k}} \times\left\{0,1, \ldots, p_{k}\right\}, F_{k}, \hat{\varphi}_{k}\right)
$$

is equivalent to the increasing inverse system $\left(\chi\left(p_{k}, 1, \beta_{k}\right), \varphi_{k}\right)$ and $\omega(a, f)$ is isomorphic to the inverse limit of the given increasing inverse system. This provides us with a useful way to study these inverse limits.

Proposition 5.4. Let $R$ be a closed invariant equivalence relation on $\omega(a, f)$. If $((x, z),(y, w)) \in R$ implies $z \neq w$, then $\omega(a, f)$ is a group extension of $\omega(a, f) / R$.

Proof. Suppose $\left(\left(x_{0}, z_{0}\right),\left(y_{0}, w_{0}\right)\right) \in R$ with $\delta=w_{0}-z_{0} \neq 0$. By minimality, for every $(x, z)$ there exists a unique $\varphi_{1}(x, z)$ such that

$$
\left((x, z),\left(\varphi_{1}(x, z), z+\delta\right)\right) \quad \in R .
$$

The graph of $\varphi(x, z)=\left(\varphi_{1}(x, z), z+\delta\right)$ is

$$
R \cap\{((x, z),(y, w)): z-w=\delta\}
$$

and is thus closed. It follows that $\varphi$ is an automorphism of $\omega(a, f)$ and $((x, z), \varphi(x, z)) \in R$ for all $x$ and $z$. Moreover, since we began with an arbitrary point of $R$, there exists a group $G$ of automorphisms such that

$$
R=\{((x, z), \varphi(x, z)): \varphi \in G\}
$$

The mapping $\varphi$ to $\delta$ provides a (group) isomorphism of $G$ onto a closed subgroup of $\triangle_{a}$ and hence a compact Hausdorff topology of $G$. Moreover, it is easy to see that $G$ now acts continuously on $\omega(a, f)$ to complete the proof.

Given the increasing inverse system $\left(\chi\left(p_{k}, q_{k}, \beta_{k}\right), \varphi_{k}\right)$ for each $k$ set

$$
I_{k}=\left\{q_{k} \beta_{k}(d) / p_{k}: d \in D_{k}\right\}
$$

and observe that $I_{k} \subset I_{k+1}$ for all $k$. Letting

$$
I(a, f)=\bigcup_{k=0}^{\infty} I_{k}
$$

it is easy to use theorem 4.10 to prove the following:

Proposition 5.5. The following are equivalent:

(a) The minimal flows $\omega(a, f)$ and $\chi\left(p^{\prime}, q^{\prime}, \beta^{\prime}\right)\left\{\omega\left(a^{\prime}, f^{\prime}\right)\right\}$ are disjoint.

(b) The minimal flows $\omega(a, f)$ and $\chi\left(p^{\prime}, q^{\prime}, \beta^{\prime}\right)\left\{\omega\left(a^{\prime}, f^{\prime}\right)\right\}$ have no common factor.

(c) For all $k,\left(p^{\prime}, p_{k}\right)=1$ and $q^{\prime} \beta^{\prime}\left(d^{\prime}\right) / p^{\prime} \notin I(a, f)$. (For all $i$ and $j,\left(p_{i}^{\prime}, p_{j}\right)=1$, and $I(a, f) \cap I\left(a^{\prime}, f^{\prime}\right)=\varnothing$.)

Proposition 5.6. Two minimal flows $\omega(a, f)$ and $\omega\left(a^{\prime}, f^{\prime}\right)$ constructed in the prescribed way are isomorphic if and only if the topological groups $\Delta_{a}$ and $\Delta_{a^{\prime}}$ are isomorphic and $I(a, f)=I\left(a^{\prime}, f^{\prime}\right)$.

Proof. Assume $\omega(a, f)$ and $\omega\left(a^{\prime}, f^{\prime}\right)$ are isomorphic. Using [6, corollary 2.7] it is straightforward to show that the proximal relation of $\omega(a, f)$ is dense in $\left\{((x, z),(y, z)): x, y \in X^{D}\right\}$ for each $z \in \Delta_{a}$. It follows that the maximal equicontinuous factor of $\omega(a, f)$ is $\left(\Delta_{a}, 1\right)$ and the topological groups $\Delta_{a}$ and $\Delta_{a^{\prime}}$ are isomorphic (see [4, Chapter 4]). 
For convenience we will assume throughout the proof that $q_{k}=1=q_{k}^{\prime}$ for all $k$. Fix $d^{\prime} \in D^{\prime}$. Then $d^{\prime} \in D_{k}^{\prime}$ for some $k$ and $\chi\left(p_{k}^{\prime}, 1, \beta_{k}^{\prime} \mid\left\{d^{\prime}\right\}\right)$ is a factor of $\omega(a, f)$, and $p_{k}^{\prime}$ divides $p_{i}$ for some $i$. Without loss of generality we can assume $p_{k}^{\prime} \mid p_{0}$. Then $\left(X, \beta_{k}^{\prime}\left(d^{\prime}\right)\right)=\chi\left(1,1, \beta_{k}^{\prime}\left(d^{\prime}\right)\right)$ is a factor of $\left(X^{D} \times \Delta_{a}, F^{p_{k}^{\prime}}\right)$. The latter is not minimal, but its minimal subsets are all isomorphic to the inverse limit of $\chi\left(p_{j} / p_{k}^{\prime}, 1, \beta_{j}\right)$ which is some $\omega(b, g)$. We can now apply proposition 5.5 to conclude that there exists $i$ and $d \in D_{i}$ satisfying $\beta_{k}^{\prime}\left(d^{\prime}\right)=\beta_{i}(d) /\left(p_{i} / p_{k}^{\prime}\right)$ or

$$
\beta_{i}(d) / p_{i}=\beta_{k}^{\prime}\left(d^{\prime}\right) / p_{k}^{\prime} \text {. }
$$

Therefore, $I\left(a^{\prime}, f^{\prime}\right) \subset I(a, f)$ and by the symmetry of the argument we have equality.

For the converse, we index the common $I($,$) set, say \left\{r_{1}, \ldots, r_{n}, \ldots\right\}$ and construct a third inverse system whose limit is isomorphic to both $\omega(a, f)$ and $\omega\left(a^{\prime}, f^{\prime}\right)$. Let $E_{k}=\{1,2, \ldots, k\}$. Starting with $r_{1}$ we can find $i(1)$ and $j(1)$ such that $p_{i(1)}$ divides $p_{j(1)}^{\prime}$ and

$$
\beta_{i(1)}\left(d_{i(1)}\right) / p_{i(1)}=r_{1}=\beta_{j(1)}^{\prime}\left(d_{j(1)}^{\prime}\right) / p_{j(1)}^{\prime}
$$

for some $d_{i(1)} \in D_{i(1)}$ and $d_{j(1)}^{\prime} \in D_{j(1)}^{\prime}$. Define $\gamma_{1} \in \mathbb{Z}^{E_{1}}$ by

$$
\gamma_{1}(1)=\beta_{i(1)}\left(d_{i(1)}\right) \text {, }
$$

and check that $\chi\left(p_{i(l)}, 1, \gamma_{1}\right)$ is a factor of both $\chi\left(p_{i(1)}, 1, \beta_{i(1)}\right)$ and $\chi\left(p_{j(1)}^{\prime}, 1, \beta_{j(1)}^{\prime}\right)$.

For the next step choose $i(2)>i(1)$ and $j(2)>j(1)$ such that $p_{j(2)}^{\prime}$ divides $p_{i(2)}$ and

$$
\beta_{i(2)}\left(d_{i(2)}\right) / p_{i(2)}=r_{2}=\beta_{j(2)}^{\prime}\left(d_{j(2)}^{\prime}\right) / p_{j(2)}^{\prime} \text {. }
$$

Notice that we still have

Define $\gamma_{2} \in \mathbb{Z}^{E_{2}}$ by

$$
\beta_{i(2)}\left(d_{i(1)}\right) / p_{i(2)}=r_{1}=\beta_{j(2)}^{\prime}\left(d_{j(1)}^{\prime}\right) / p_{j(2)}^{\prime} .
$$

$$
\gamma_{2}(n)=\beta_{j(2)}\left(d_{j(n)}\right), \quad n=1,2 .
$$

Then $\chi\left(p_{j(2)}^{\prime}, 1, \gamma_{2}\right)$ is a factor of both $\chi\left(p_{i(2)}, 1, \beta_{i(2)}\right)$ and $\chi\left(p_{j(2)}^{\prime}, 1, \beta_{j(2)}^{\prime}\right)$. In addition, there is an increasing homomorphism

$$
\theta_{1}: \chi\left(p_{j(2)}, 1, \gamma_{2}\right) \rightarrow \chi\left(p_{i(1)}, 1, \gamma_{1}\right)
$$

This alternating construction can be repeated infinitely often to produce an increasing inverse system. Obviously, the inverse limit $\omega(b, g)$ is a factor of both $\omega(a, f)$ and $\omega\left(a^{\prime}, f^{\prime}\right)$. To complete the proof, one checks that these maps separate points.

To conclude we apply the previous results to our study of the dynamics of weak mixing flows of the form $\left(X^{D}, U(\pi, \alpha)\right)$.

THEOREM 5.7. Let $(X, T)$ have TMSJ \{PTMSJ\}, let $\pi$ and $\pi^{\prime}$ be compact permutations of the countable sets $D$ and $D^{\prime}$, and let $\alpha$ and $\alpha^{\prime}$ be elements of $\mathbb{Z}^{D}$ and $\mathbb{Z}^{D^{\prime}}$ such that $U(\pi, \alpha)$ and $U\left(\pi^{\prime}, \alpha^{\prime}\right)$ are weakly mixing. Then

(a) there exists a minimal set $\bar{M}_{0}$ of $\left(X^{D}, U(\pi, \alpha)\right)$ which is a group extension of every other minimal subset of $\left(X^{D}, U(\alpha, \pi)\right)$;

(b) the $U(\pi, \alpha)$ minimal sets are dense in $X^{D}$;

(c) minimal subsets $M$ and $M^{\prime}$ of $\left(X^{D}, U(\pi, \alpha)\right)$ and $\left(X^{D^{\prime}}, U\left(\pi^{\prime}, \alpha^{\prime}\right)\right)$ respectively are disjoint if and only if they have no common factor except the trivial flow. 
(d) if $\left(X^{D}, U(\pi, \alpha)\right)$ and $\left(X^{D^{\prime}}, U\left(\pi^{\prime}, \alpha^{\prime}\right)\right)$ are isomorphic, then the orders of $\pi$ and $\pi^{\prime}$ are equal and

$$
\operatorname{Ave}(\pi, \alpha)=\operatorname{Ave}\left(\pi^{\prime}, \alpha^{\prime}\right)
$$

Proof. (a) Use theorem 3.4, corollary 4.7, proposition 5.3, and proposition 5.5

(b) It suffices to carry out the proof for $\pi$ with finite order which can easily be done using propositions $2.2,2.3$ and 2.7 .

(c) Use theorem 4.10 and proposition 5.5.

(d) First observe that the orders of $\pi$ and $\pi^{\prime}$ must both be finite or must both be infinite because a $\chi(p, q, \beta)$ has only finitely many finite factors while $\omega(a, f)$ has infinitely many. The finite order case follows from theorem 4.11. For the infinite order case, first check that if $\omega(a, f)$ is the inverse limit of $\left(\chi\left(\rho_{k}, 1, \lambda_{k}\right), \varphi_{k}\right)$ as constructed in the beginning of the section, then $I(a, f)=$ Ave $(\pi, \alpha)$. Now apply proposition 5.6.

The author is indebted to the referee for some incisive observations which were used to simplify and reorganize parts of this paper.

The author was supported by N.S.F. Grant MCS-8101708.

\section{REFERENCES}

[1] R. V. Chacon. Weakly mixing transformations which are not strongly mixing. Proc. Amer. Math. Soc. 22 (1969), 559-562.

[2] A. del Junco, A family of counter-examples in ergodic theory. Preprint.

[3] A. del Junco, M. Rahe \& L. Swanson. Chacon's automorphism has minimal self-joinings. J. Analyse Math. 37 (1980), 276-284.

[4] R. Ellis. Lectures on Topological Dynamics. W. A. Benjamin: New York, 1969.

[5] H. Furstenberg, H. Keynes \& L. Shapiro. Prime flows in topological dynamics. Israel J. Math. 14 (1973), 26-38.

[6] H. Keynes. The structure of weakly mixing minimal transformation groups. Illinois J. Math. 15 (1971), 475-489.

[7] H. Keynes \& D. Newton. Real prime flows. Trans. Amer. Math. Soc. 217 (1976), $237-255$.

[8] N. Markley. Characteristic sequences. Recent Advances in Topological Dynamics. Lecture Notes in Mathematics vol. 318. Springer-Verlag: Berlin, 1972.

[9] D. Rudolph. An example of a measure preserving map with minimal self-joinings, and applications. J. Analyse Math. 35 (1979), 97-122. 